\title{
COGNITIVE APPROACH TO DISTANCE LEARNING MATERIALS PREPARATION ADAPTED TO ASYNCHRONOUS MODE
}

\author{
Oleg Zaikine, Emma Kushtina, Przemyslaw Rozewski \\ Technical University of Szczecin, Faculty of Computer science and Information Technology, \\ Żotnierska 49, 71-210 Szczecin, Poland, Tel: (+4891) 44956 64, Fax: (+4891) 4876439 \\ \{ozaikine, ekushtina,prozewski\}@wi.ps.pl
}

Received 3 April, 2003

\begin{abstract}
The authors discuss the method of designing the distance learning didactic materials for the asynchronous mode. The approach is based on the modular structure of the fundamental knowledge. The new role of the teacher is being discussed as well as its impact on the didactic materials' structure and content. The paper introduces the knowledge representation model designed to meet the requirements of theoretical knowledge presentation in a selected domain using the Learning Object concept.
\end{abstract}

Keywords: distance learning, knowledge representation model, didactic materials

\section{Introduction}

The continuous process of expansion of the professional knowledge, wide development of virtual organizations and wide spread of the world area network, are the reasons for implementation of the Distance Learning (DL) systems in corporation environment. The issue of a long-life and continuous education obliges the corporations to improve their educational programs towards the DL modules. Nowadays the employee of an organization has to enlarge his/her professional knowledge because of continuous update on technological knowledge, global market competition and wide usage of the new High-Tec technologies. The main vendors issued last version of their LMS/LCMS with a mechanism and tools specially designed to meet corporation requests.

From the implementation and maintenance costs' point of view the asynchronous mode is the most effective one for organizing DL in schools, universities and enterprises. The learning process in asynchronous mode is made of several sub-processes: administration, education and organization. A more detailed analysis shows the different role of the teacher in conventional teaching and distance learning. It means that the methods of conventional teaching cannot be directly used in the telecommunication space [6]. In traditional education the teacher has the opportunity to communicate with the student and to control the dialog over the lesson's subject. In DL the student explores the didactic material on his/her own in order to achieve the satisfactory level of knowledge. The teacher is no longer directly involved in the educational process, he/ she is only responsible for the student's knowledge validation procedure and the essential consultation.

The analysis of existing models of knowledge representation shows that none of them satisfy the demands of DL. It is shown in [8] that the structure of knowledge, which differs from the Rules Systems, can be presented on the basis of Semantic Networks (SN). SN can represent both abstract categories and certain objects. The big obstacle for using the SN is the difficulty with formal representation of the semantic relation. The SNs are the starting point for such knowledge representation models as Mind Maps [2], Conceptual Maps [10] and Topic Maps [5]. Each model 
is oriented towards a specific kind of knowledge and a concrete user. Each of them is dedicated to specific kind of tasks and a specific domain. The level of universality in DL knowledge model should be high. The appropriate model must at least work with one kind of knowledge (e.g. theoretical) in all of its contexts. The final and formalized knowledge representation system should merge the representation of the domain knowledge with the issue of a corresponding pedagogical approach, which are used to learn about a subject, and the knowledge manipulation language.

\section{Usage of the black box principle for modelling a learning / teaching process}

Targets of the DL are automation of information exchange processes and an intentional formation of knowledge in the educational process. In the conceptual DL model we don't examine the internal representation of knowledge and coding of 'gestalts'. We consider each participant of the DL process as a source and a receiver of information as well as an operator in the generalized information space. Using the principle of the black box we examine the didactic materials and the teacher's skill at input and the student's acquired/perceived knowledge at output of a DL process. Therefore, in the context of information, the model of a DL process represents the relation between the input information, given by the teacher, and the knowledge acquired by the student.

The necessary condition for this model's existance is usage of an identical language for the "input" and "output" of the DL process. The performance of this condition in conventional education gives the teacher the opportunity for a direct control of the educational process, using qualitative criteria. In turn, a subsequent analysis of the acquired knowledge evaluation gives the possibility to correct the long-term and short-term planning of the educational process. Using these plans the teacher can improve the semantic expressiveness of didactic materials by means of changes in the sequence and depth of the examined phenomena/concepts, selection of metaphors, setting of associative links, description of the concept in different contexts.

The unambiguity of the knowledge perception is being analyzed by the teacher during the interaction with the students. Although the long-term planning for the distance and conventional education is identical in many acpects, the scheduling and the operative control of the plan's performance will differ considerably. It is caused by the fact that in DL the teacher has no direct contact with the student but communicates with $\mathrm{him} / \mathrm{her}$ in a non-conventional environment.

\section{Relation between the knowledge sources, the learning environment and the knowledge representation language}

The natural language (NL) is the basic mode of knowledge representation and transfer in conventional teaching. However, various types of symbolic/artificial languages can also be used, what gives additional possibilities to enhance the semantic expressiveness of the NL. It is applies equally to lectures and for didactic materials. From the point of view of ergonomics and psychology, the computer environment sets bounds on the NL usage. On the other hand, the computer environment gives unlimited possibilities for using symbolic and artificial languages and media.

The matter is how to change the direct 'teacher-student' contact based on NL into a dialogue in the telecommunication space which uses a symbolic/artificial language, without losing the basic semantic content of knowledge. The teacher is not the only source of knowledge, that uses unlimited NL, the books, the media, the environment play the same role. Therefore, the language of knowledge representation in DL must correspond with the NL and languages of other sources of knowledge. In other words, the new knowledge obtained from the external sources has to be easly embedded in the conceptual system of educational process.

\section{Structure as a tool for analysing the information content}

The notions of the structure and information are inseparable in the processes of teaching and communication. A man is surrounded by the continual information flow. The matter is what we receive and how we use it. The structure is a tool, which conduces to the analysis and exposure of the sense and content of information. Only after this analysis can we estimate the utility of information. The structures are embodied as designs basing on the vocabulary and grammar of the formalized language (FL). The degree of the language formalization can vary from NL to simple operations for designing graphic schemes. The formalized language seen as a tool for the knowledge representation requires a definition of elements and operations/rules for determining structures. The more elaborate structures, the more expressive force of language, which is used for their construction.

Automation of the construction of complex structures on the basis of atomic elements demands a formal definition of operations. If some structures can be constructed in different ways, then the operations of 
the FL can lead to an ambiguous 'perusal' of the structure's sense. Therefore, the level of the structures' complexity and variety of their 'perusal' define the semantic expressiveness of the FL. Reducing the number of atomic elements and operations/rules for the structure's construction we provide a higher level of FL semantic interpreting. Therefore, the semantic expressiveness and un-ambiguity depend on the formalization level of the used language in an opposite way. The FL describes the basic concepts and scenarios necessary for the planned dialogue and collective purposive behaviours.

\section{Theoretical and procedural knowledge}

The knowledge as an object and purpose of study can be divided into two basic kinds: fundamental (theoretical) and operational (procedural). The fundamental knowledge reflects the conceptual thinking and contains new paradigms, problems statements, principles of behaviour etc. Procedural knowledge is necessary for the development and realization of scenarios, algorithms, the performance of various kinds of operations. The analysis of various problems and situations, examined in the educational process, demands a simultaneous use of both kinds of knowledge but in different proportions, depending on the complexity of the problem that is being solved. New methods of the procedural knowledge representation and DL technologies allow creating effective systems of computer training (simulators, expert systems, etc). Such systems set relationship between practical skills (speed, accuracy, conformity to standard) and available theoretical base in real time.

On the other hand, the conceptual thinking is shown in the following: the abilities of abstraction and generalization, setting associative links, inference of a new knowledge on the basis of the available one, formulation of paradoxical knowledge which exceeds the existing paradigm. There are no objective quantitative methods of evaluating the conceptual knowledge, acquired during training. Therefore, the assessment of the quality of learning conceptual knowledge is based on objective properties of the domain and techniques of studying it. With the same amount of knowledge in the subject domain and other equal conditions the various techniques of training can affect the development of conceptual thinking in a different degree. The responsibility for the choice of the training technique lies in the hands of the teacher. Therefore, in the conditions of DL it is necessary to use the methods of theoretical knowledge representation, that give opportunities to present objective knowledge in a subject domain as well as techniques of studying it.

\section{Concept as a structure of storing knowledge}

The reality is defined through the unlimited and diverse set of information or stimulus, which is absorbed by man. The cognitive science assumes the mind's natural ability to categorise incoming information and knowledge. The mind stores the outcomes of the incoming flow in the form of a concept. The concept is considered as a structure of mind representation, which consist of the proper description of a singlemeaning class [7]. The methods of teaching conceptual knowledge take an advantage of the concept's communication level between the students. The teacher can use metaphors or other pedagogical methods, the most important issue is establishing the cohesion state what is achieved when both, teacher's and student's minds refer to the same concept prototypes simultaneously $[9,12]$.

The knowledge representation model consists of: a definition of the semantic unit, related unit operations and appropriate description language. The DL environment does not give the opportunity to use the natural language as a knowledge carrier in the regular way. This premise causes the need of a new, special language development. In terms of the problems discussed above the model of storing and exchanging knowledge should:

Correspond with the natural language and other languages of knowledge representation.

- Ensure a sufficient level of structure legibility.

- Ensure a sufficient level of clarity, which allows the language to create sophisticated structures.

- Ensure the possible, future ability to change the concept's content according to the context.

- Ensure the ability to create a complex structure basing on common ones.

- Ensure the ability to merge separate structures into a Semantic Network with a limited semantic relation.

- Ensure the ability to join the domain knowledge and pedagogical methodology of teaching it, into one model.

The basic element of the representation language is a logical unit, which in case of cognitive science is considered as a concept. Every concept is a representation of some class of objects characterised by common properties, every set of concepts can be written down as a matrix. The number of rows represents the number of common attributes of the objects and the number of columns symbolizes the amount of objects merged into one class. The concept's depth in the 
specified domain is equivalent to the number of elements in the matrix.

A fully functional language should contain a mechanism of concept manipulation. The manipulation mechanism includes methods to broaden the semantic of the concept, which means that the new attribute, new object's description or semantic relations can be added to the semantic. Every domain can be covered and formalized in the form of a graph based on the described above language. The nodes of the graph are the concepts and the links between them represent semantic relations.

The DL successfully adapts the idea of the concept to the telecommunication space. A single operation of the Learning Object (LO), encapsulated part of knowledge, creation gives profit by the possible future multiple usage of the learning object in many perspectives and backgrounds. The task of the LMS/LCMS systems is to manage the learning objects, which are stored in repositories. The structure and construction of the learning object are covered in detail by the SCORM standard. There is a lack of information or knowledge about the content of the learning object. The international research society has investigated the problem of the learning object for several years $[3,4,11]$, however, the general solution has not been found. Until now, the set of guidelines and rules has been published [4].

\section{The method of knowledge domain description based on the algorithm of concept network creation}

The goal of the first phase of the didactic material preparation is to identify the knowledge in a specific subject domain. The subject matter expert basing on the algorithm of creating the conceptual network (Fig. 1) localizes the concepts adequate for the specified domain, merges the concepts according to their semantic meaning and creates a multimedia representation of each of them. The algorithm covers the domain the knowledge was extracted from. The results are compatible with the SCORM standard.

The subject domain's analysis is conducted on the first step of the expert's work. The subject matter expert examines the domain and the concepts are collected according to the rules of concepts' description. The identified set of concepts is enhanced by adding the semantic relation. Simultaneously the formulation of the concepts multimedia representation is being performed. As a result of the algorithm each concept is represented as a data conglomerate being the multi- media description (metaphor) of the given concept. The main function of each metaphor is to widely describe the concept or the idea, which is covered by the concept. The abstract form of the concept implies digital representation. The completely processing information, including concepts, meta-information (each notion is characterised by author, data, etc.), media files, is stored in the repository. The Criterion I of the conceptual network algorithm decides whether the choice of the multimedia metaphor is correct. The expert, who is carefully considering the judgement process, can enlarge the depth of the concept's metaphor basing on his/ her knowledge of the subject. After the positive semantic verification of concepts, the network of concepts is applied to the repository structure. The whole system was evaluated again according to criterion II. The network of concepts should cover every bit of knowledge in the specified domain.

The results of the algorithm are: (a) the concept semantic network, (b) the repository, (c) the hypermedia network of concepts, which maps the concepts on their multimedia representations that are in the repository. The algorithm's outcomes are fully compatible with the SCORM standard. The repository stores XML files and a whole spectrum of media files. The relations at the semantic level and multimedia reflections are converted to XML structure based on the SCORM standard.

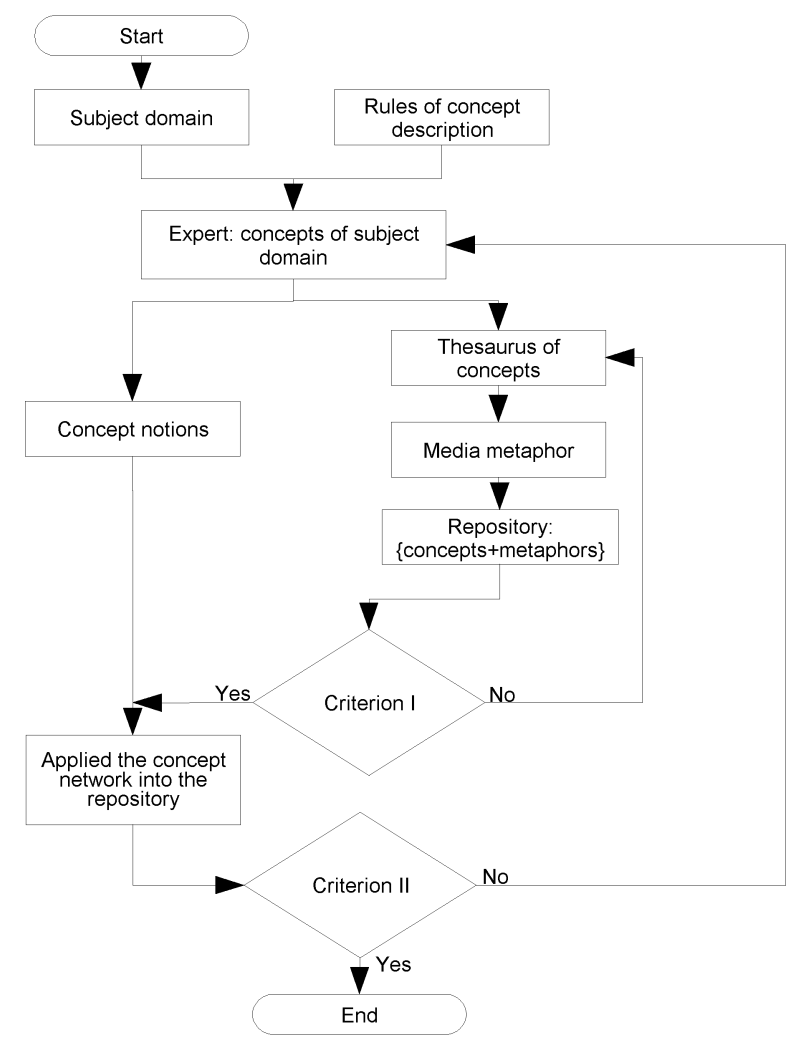

Fig. 1. The algorithm of creating the Conceptual Network 
The didactic materials' compilation algorithm (Fig. 2) adapts the didactic materials for the student by taking under consideration the general educational standards and pedagogical conditions of the learning process. The goal of the algorithm is to develop a sequence of educational elements, which is delivered to the student through the telecommunication network. The algorithm is based on the network of concepts, repository and student's profile. The described below method can be easily adapted for existing LMS/LCMS systems.

Based on prior information about the student's base knowledge in the subject area, the network of concepts is organised. After the procedure is completed a set of basic (initial) concepts is selected and their relationship with the other ones is determined. The result of this procedure is a hierarchical multilevel graph with basic concepts at top level. The concepts at low level of the hierarchical graph are interpreted as objectives of learning. Such graphic representation of knowledge determines uniqueness of each concept and the number of its semantic links. The links define semantic richness of each concept and its importance for other concepts.

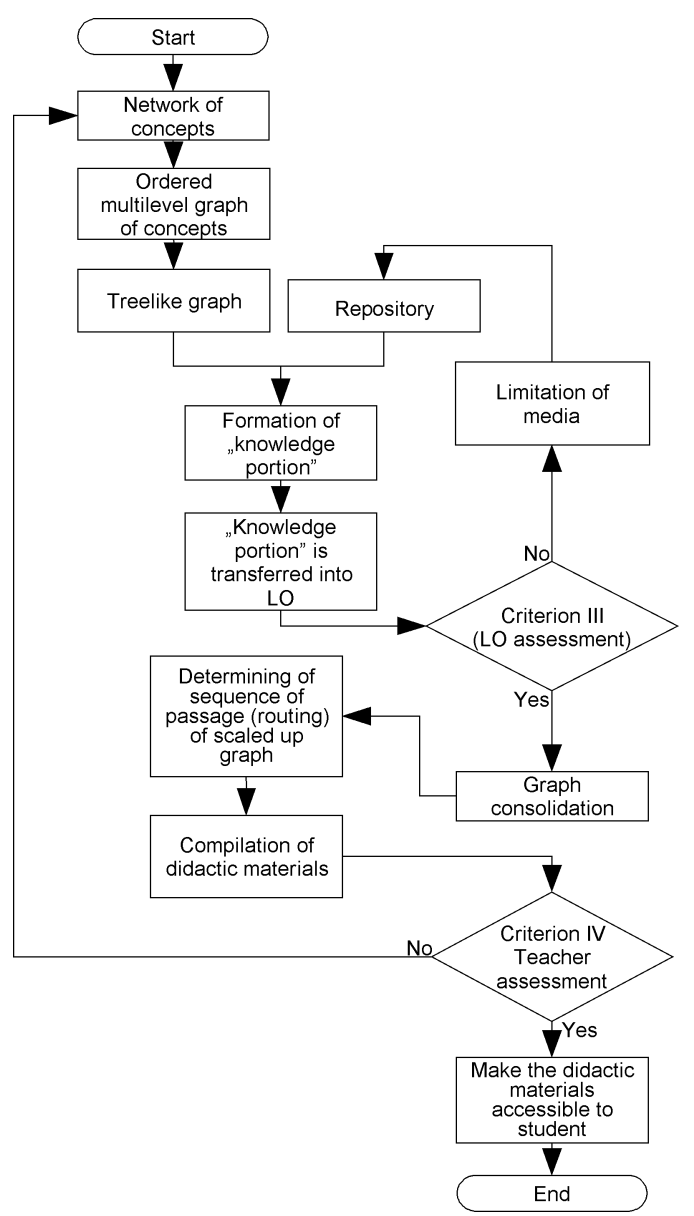

Fig. 2. The didactic materials' compilation algorithm
At the next stage of the algorithm, the multi-level hierarchical network is decomposed to its own set of sub-graphs, the concepts included in a single subgraph are not connected with the other ones. After the decomposition, every sub-graph is transferred into the tree-like graph. The roots of each tree represent the final educational goal. The transformation of subgraphs into trees may use the duplicating operation, since the concept could be multi-related in the selected sub-graph. The multi-related concept is duplicated to achieve the correct meaning of every context in the tree. The trees are covered by the 'knowledge portion', which is constructed basing on cognitive regulations $[1,7]$. The selected 'knowledge portion' is a basic element of the Learning Object (LO). Transformation of the 'knowledge portion' into a LO demands a representation of the concept in the repository content.

The data and its metadata are analyzed basing on criterion III. The criterion III estimates the 'size' of the data according to the network's bandwidth limitation, the copyright issue and the resources accessibility. Every student can be distinguished due to his/her cognitive learning style. The 'knowledge portions', when aiming at the best effectiveness, should show up one at a time. The consolidation process joins the concepts of every 'knowledge portion' into one node. The knowledge incorporated into the 'knowledge portion' together with the essential metadata description creates the Learning Object. The generated LOs are impinged on the concepts' network, which is after that converted to a LOs network. Afterwards the sequence of nodes (LOs) is set. The established LOs' chain determines the way of learning of each student. Before the LOs' sequence will be placed in the repository, all elements are converted to a SCORM-compatible form. The course instructor/teacher has to make the final decision about the didactic materials' acceptation. Finally, the didactic materials are accessible for every registered student.

\section{Summary}

The fundamental and procedural knowledge requires different learning environments. However, the learning process takes advantages of the cooperation of both types of knowledge. The proportion of the knowledge involved in the current learning process depends on the learning objectives. The efficient learning system must learn the way to use both types of knowledge effectively.

The world-wide DL collaboration platform is the main reason for the new DL standard. The DL standard 
guarantees [9]: (a) durability - does not require modification as the version of system software changes, (b) interoperability - operates across a wide variety of hardware, operating systems and Web browsers, (c) accessibility - can be indexed and found when need, (d) reusability - can be modified and used by many different tools and in different contexts. The global usage of the SCORM standard allows the education society to build up a gigantic didactic materials' repository. The access to the high quality didactic material gives unlimited educating possibilities.

The presented algorithms could be used as a methodical tool for a teacher, especially in DL. The new role of the teacher implies an innovative approach to the learning processes' management. A highly profiled course delivers educational content autonomously and efficiently to every student.

The cognitive approach examined in the paper supposes joint, compatible work of the expert in a given domain, the knowledge engineer and the teacher. The most complex task in the proposed approach is designing the knowledge representation model for a given subject domain. It demands considerable efforts in knowledge mining in the given domain and only the most competent experts can solve this task, guaranteeing a high level of quality of the knowledge model. Apart from the expert, other participants of the given project, namely knowledge engineer and the teacher, have also a great impact on the quality of all distance learning processes.

\section{References}

1. Anderson J. R. Cognitive psychology and its implications, Freeman, San Francisco, 1980. 531 p.

2. Buzan T. The Mind Map Book - Radiant Thinking, BBC Books, London, 1993. $230 \mathrm{p}$.

3. Downes S. Learning objects: Resource for Distance Education Worldwide. International Review of Research in Open and Distance Learning, Vol. 2, No. 1, 2001.

4. Hamel C. J., Ryan-Jones D. Designing Instruction with Learning Objects. International Journal of Educational Technology, Vol. 3, No. 1, 2002.

5. ISO/IEC 13250, Information technology - SGML Applications - Topic Maps, International Organization for Standardization, Geneva, 2000.

6. Kushtina E., Zaikin O., Enlund N. A knowledge base approach to courseware design for distance learning. In: Proceedings of the 7th Conference of European University Information Systems (EUNIS'01), Berlin, Humboldt-University, 2001.

7. Maruszewski T. Psychologia Poznania, Gdańskie Wydawnictwo Psychologiczne, Gdańsk, 2002. 428 p. (in Polish).

8. Quillian, M. Ross, Semantic Memory, in M. Minsky, ed., Semantic Information Processing, MIT Press, Cambridge, Massachusetts, 1968.

9. SCORM - Sharable Content Object Reference Model v1.2, Advanced Distributed Learning, http://www.adlnet.org, 2001.

10. Sowa John F. Knowledge Representation: Logical, Philosophical, and Computational Foundations, Brooks Cole Publishing Co., 2000, 420 p.

11. Wagner Ellen D. The New Frontier of Learning Object Design. The eLearning Developers' Journal, June, Vol. 18, 2002.

12. Wiley D. A. (ed.). The Instructional Use of Learning Objects. Association for Educational Communications and Technology, 2002. 